\title{
Investigation of carbapenemases and aminoglycoside modifying enzymes of Acinetobacter baumannii isolates recovered from patients admitted to intensive care units in a tertiary-care hospital in Brazil
}

\author{
Milena Polotto ${ }^{[1],[2], ~ T i a g o ~ C a s e l l a ~}{ }^{[2]}$, Fernanda Modesto Tolentino ${ }^{[1],[2],}$ \\ Mayra Mioto Mataruco ${ }^{[3]}$, Naiady Konno Madela Porto ${ }^{[2]}$, \\ Mirella Fontana Batista Binhardi ${ }^{[1]}$ and Mara Corrêa Lelles Nogueira ${ }^{[2]}$
}

\begin{abstract}
[1]. Instituto Adolfo Lutz, Centro de Laboratório Regional de São José do Rio Preto, São José do Rio Preto, SP, Brasil. [2]. Faculdade de Medicina de São José do Rio Preto, Centro de Investigação de Microrganismos, São José do Rio Preto, SP, Brasil.

[3]. Universidade Federal de São Carlos, São Carlos, SP, Brasil.
\end{abstract}

\begin{abstract}
Introduction: Acinetobacter baumannii are opportunistic bacteria, highly capable of acquiring antimicrobial resistance through the production of carbapenemases and aminoglycoside modifying enzymes (AMEs). Methods: Carbapenemase and AME genes were investigated in A. baumannii recovered from inpatients of a Brazilian hospital. Results: The key genes found were $b l a_{\text {OXA-51- }}$ like, the association ISAba1- bla $a_{\mathrm{OXA-23-like}}$, and the AME genes $a p h\left(3^{\prime}\right)-V I$, $a a c\left(6^{\prime}\right)-I b$, aac(3)-Ia, and aph (3')-Ia. Different clusters spread through the institution wards. Conclusions: The dissemination of bla $\mathrm{OXA}_{\mathrm{O}-23-\mathrm{like}}$ and AME-carrying A. baumannii through the hospital highlights the need for improved preventive measures to reduce the spread of infection.
\end{abstract}

Keywords: Acinetobacter baumannii. Carbapenemases. Aminoglycoside modifying enzymes.

Acinetobacter baumannii are opportunistic pathogens that cause nosocomial infections mainly in immunocompromised patients and those admitted to intensive care units (ICUs). This microorganism is highly capable of surviving and spreading through hospital environments and acquiring resistance to multiple classes of antimicrobials ${ }^{1}$. A. baumannii is considered to be one of the six particularly problematic pathogens in terms of antimicrobial availability issues arising from drug resistance, and its multidrug-resistant clones have spread globally ${ }^{1}$.

Carbapenem antibiotics have been widely used for treating A. baumannii infections; however, the emergence of resistant strains has been increasingly reported and is often associated with high mortality rates and a substantial increase in hospitalization costs ${ }^{2}$. Moreover, as carbapenem-resistant (CR) A. baumannii also presents resistance to other antimicrobial

Corresponding author: Mara C.L. Nogueira.

e-mail: ml.nogueira@famerp.br

Orcid: 0000-0002-6070-7716

Received 1 March 2019

Accepted 13 August 2019 classes, alternative therapeutic approaches need to be explored ${ }^{3}$. The most commonly identified mechanism of carbapenem resistance among $A$. baumannii isolates is the production of oxacillinases (OXAs), which include OXA-23-like, OXA-24like, OXA-58-like, and the intrinsic OXA-51-like enzymes ${ }^{4}$.

Aminoglycosides are a potential treatment option for $\mathrm{CR}$ A. baumannii infections ${ }^{3}$; however, several isolates are capable of producing aminoglycoside-modifying enzymes (AMEs), the most important aminoglycoside resistance mechanism in A. baumannii ${ }^{5}$. Information about aminoglycoside resistance and the prevalence of AME-producing A. baumannii in Brazil is scarce, and few studies have been conducted to investigate these enzymes in this species.

Thus, the objectives of this study were to investigate the prevalence and diversity of carbapenemases and AME-encoding genes in CR A. baumannii isolates, and to evaluate the clonal relationships among them.

The study was conducted from July to December 2012 at a 746-bed public tertiary-care teaching hospital in São José do Rio Preto (São Paulo State, Brazil). The hospital receives patients from more than 100 cities in this region and has 117 ICU beds. 
One hundred non-duplicated CR A. baumannii isolates were obtained from different clinical specimens from patients admitted to 6 different hospital ICUs (Cardiology-ICU, Emergency-ICU, General private-ICU, General publicICU, Pediatric-ICU, and Semi-intensive-ICU). Initially, identification of the Acinetobacter baumannii-Acinetobacter calcoaceticus complex was performed using the VITEK-2 Compact system (bioMérieux, Marcy-l'Étoile, France). For species confirmation, a duplex-polymerase chain reaction (PCR) was used to detect the specific intergenic region present in A. baumannii. Primers (5'-CATTATCACGGTAATTAGTG and 5'-AGAGCACTGTGCACTTAAG) were used to amplify a specific internal $208 \mathrm{bp}$ fragment from the Intergenic Specific region of A. baumannii, and a highly conserved $425 \mathrm{bp}$ region of the recA gene was used as a control ${ }^{6}$ (5'-CCTGAATCTTCTGGTAAAAC and 5'-GTTTCTGGGCTGCCAAACATTAC).

Antimicrobial susceptibility testing was performed by microdilution using the VITEK-2 Compact system for the following antimicrobials: ceftazidime, cefepime, meropenem, gentamicin, ciprofloxacin, and tigecycline. The disc-diffusion method was used to test imipenem, piperacillin/tazobactam, and amikacin susceptibility, and the broth microdilution method, Policimbac (Probac, São Paulo, Brazil), was used to test polymyxin B susceptibility. The results were analyzed according to the Clinical and Laboratory Standards Institute (CLSI) breakpoints (2014).

For DNA extraction, colonies of a recent pure culture (up to 24 hours) grown on MacConkey Agar plates were suspended in $500 \mu \mathrm{l}$ of sterile water in a $1.5 \mathrm{ml}$ tube, homogenized, and incubated at $95-99^{\circ} \mathrm{C}$ for 10 minutes. Next, this mixture was placed on ice for 3-5 minutes centrifuged at $16.1 \mathrm{rcf}$ for 3 minutes; the supernatant was collected for PCR analysis. The carbapenemase genes $b l a_{\text {OXA-51-like, }}, b l a_{\text {OXA-23-like }}, b l a_{\text {OXA-58-like, }}$ $b l a_{\text {OXA-24-like, }}, b l a_{\text {OXA-143-like, }}, b l a_{\text {OXA-48-like, }}, b l a_{\text {IMP }}, b_{\text {VIM }}, b l a_{\text {SPM }}$, and $b l a_{\mathrm{KPC}}$, and the insertion sequence ISAbal upstream of $b l a_{\mathrm{OXA}}$ ${ }_{23-\text {-like, }}$ were all screened and sequenced using a 3130 Genetic Analyzer (Thermo Fisher Scientific). Three multiplex-PCRs were performed, as described previously ${ }^{5}$, to investigate the following AME genes: one triplex-PCR intended to amplify $a a c(3)-I a$, $a a c(3)-I I a$, and $a a c\left(6^{\prime}\right)-I h$, another triplex-PCR to amplify $\operatorname{aph}\left(3^{\prime}\right)-V I$, ant(2')-Ia, and the internal control $r r n$ gene, and a duplex-PCR to amplify aph(3')-Ia and $a a c\left(6^{\prime}\right)-I b$. All the AME genes detected were confirmed by sequencing. Conventional single PCRs, as described previously ${ }^{7}$, were used to screen the $16 \mathrm{~S}$ rRNA methyltransferase genes arm $A$ and $r m t B$. Molecular relationships between the isolates carrying AME genes were evaluated by repetitive element sequence-based PCR (REP-PCR). Patterns were analyzed with BioNumerics ${ }^{\circledR}$ software version 6.1 (Applied Maths, Saint-Martens-Latem, Belgium), using the Dice band-based similarity coefficient and the unweighted pair group method with arithmetic mean as clustering methods. Isolates were assigned to the same cluster if their similarity coefficient was $\geq 90 \%$.

All $A$. baumannii isolates displayed a multidrug-resistant phenotype; in addition to carbapenems, isolates showed resistance to piperacillin/tazobactam, cefotaxime, ceftazidime, cefepime, and ciprofloxacin. The resistance rates to amikacin, gentamicin, tigecycline, and polymyxin B were $76 \%, 32 \%$, $12 \%$, and $2 \%$, respectively. Bacterial isolates originated from tracheal aspirate $(52 \%)$, catheter tips $(22 \%)$, urine $(8 \%)$, blood $(5 \%)$, biopsy (4\%), secretions ( $3 \%$ ), pleural fluid ( $2 \%)$, and other clinical specimens (sputum, cerebrospinal fluid, bronchoalveolar lavage, and bone marrow; $1 \%$ each). The isolates recovered from respiratory tract samples corresponded to $54 \%$ of collected CR A. baumannii. This species is an important agent related to mechanical ventilator-associated pneumonia (VAP), contracted from colonized patients, environmental surfaces, and other unknown sources ${ }^{8}$. In the present study, the respiratory tract was the most important source of CR A. baumannii, which is in accordance with several other countries ${ }^{9}$. The second greatest source of bacteria was catheter tips, which could be indicative of bloodstream infections; or contamination, as previous study reportedy ${ }^{10}$

All the CR A. baumannii were positive for bla $a_{\text {OXA-51-like }}$ and $b l a_{\text {OXA-23-like }}$ genes, and the detection of bla $a_{\text {OXA-51-like }}$ confirmed the species identity in all isolates ${ }^{11}$. High prevalence and dissemination of bla $a_{\mathrm{OXA}-23}$ in $A$. baumannii have been reported worldwide, including Brazil ${ }^{11,12}$. Moreover, all isolates harbored the ISAbaI upstream of bla $a_{\text {OXA-23-like }}$ gene, which might be the cause of the observed carbapenem resistance ${ }^{13}$.

Among the AME-encoding genes, the most frequently detected was aph $\left(3^{\prime}\right)-V I$, present in $55 \%$ of the isolates, followed by $a a c\left(6^{\prime}\right)-I b$, detected in $47 \%$, aac (3)-Ia in $27 \%$, and $a p h\left(3^{\prime}\right)-I a$ in $22 \%$ of the isolates. All the other investigated carbapenemase and AME genes were not detected. Fifty percent of the isolates presented one AME gene, $26.7 \%$ presented two, and $24.4 \%$ presented three AME genes (Table 1).

The production of AMEs is the most important aminoglycoside resistance mechanism in A baumannii $^{3}$. In this study, four AME genes were found, and two of them, $a p h\left(3^{\prime}\right)-V I$ and $a a c\left(6^{\prime}\right)-I b$, are known to be responsible for amikacin resitance ${ }^{14}$.

The majority of the isolates $(97.2 \%)$ that harbored the $\operatorname{aph}\left(3^{\prime}\right)-V I$ gene displayed resistance to amikacin (Table 1); the high frequency of amikacin inactivation by this enzyme has been previously described ${ }^{14}$. Among the 28 isolates that harbored the gentamicin resistance gene aac(3)-Ia, 85\% were resistant to gentamicin, suggesting the production of the AAC(3)-Ia enzyme in these isolates. The high prevalence and coexistence of these AME genes circulating in the hospital environment is problematic as they limit the effectiveness of aminoglycoside usage as antimicrobials. Furthermore, these genes are highly mobile and are often transferred on plasmids, integrons, and transposons along with other resistance genes, such as $b l a_{\text {OXA-23-like, }}$ enabling the spread of several resistance genes simultaneously ${ }^{15}$.

The molecular relationship between the $86 \mathrm{CR}$ A. baumannii carrying AME genes was first elucidated by visual analysis of the dendrogram, in which the isolates were separated into 5 clusters (A-E); these were then divided into 19 sub-clusters 
TABLE 1: Frequency of aminoglycoside modifying enzymes (AMEs) genotypes, substrate specificity, and aminoglycosides susceptibility phenotypes of the carbapenem resistant $A$. baumannii.

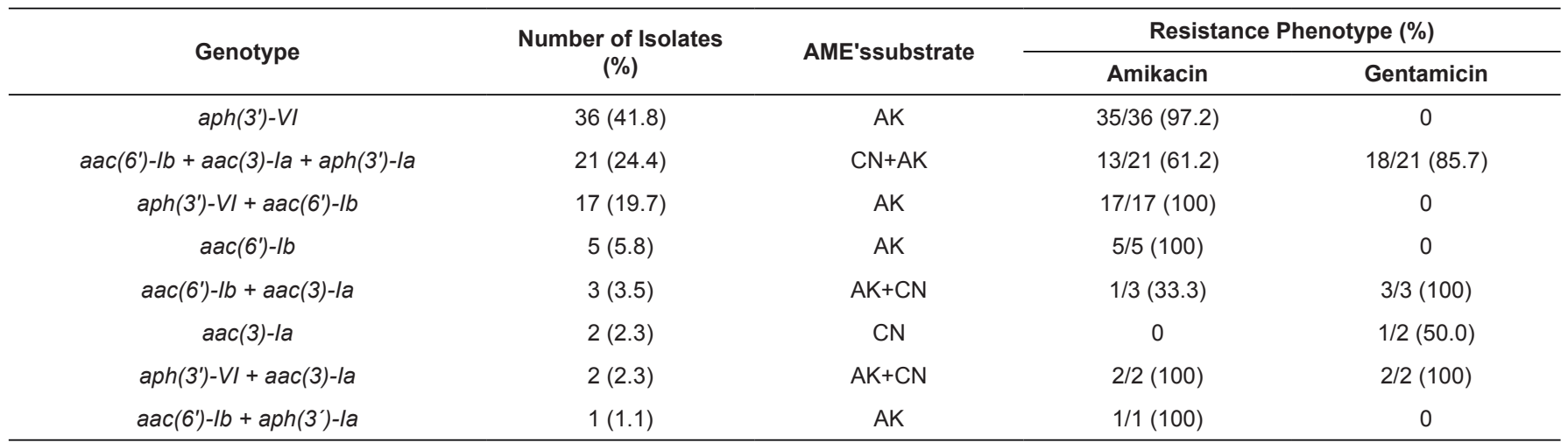

AK: amikacin; CN: gentamicin.

named A1-A3, B1-B3, C1-C5, D1-D6, E1, and E2, according to similarity coefficients $\geq 90 \%$ [Supplementary data (Figure 1)]. Eight clusters (A2, A3, B2, B3, C3, C4, C5, and D5) were considered sporadic given their duration in days and the small number of isolates [Supplementary data (Figure 1)]. Thirty four percent of the $A$. baumannii isolates belonged to cluster B1, distributed across all wards over the duration of the study, indicating an endemic cluster. The inter-ward transmission and clonal spread of the A. baumannii isolates was evidenced in some clusters like A1 (isolates Ac18, Ac47, Ac57, and Ac59), B1 (isolates Ac08, Ac09, Ac11, Ac14, and Ac17), D1 (isolates Ac04, Ac05, and Ac58), and D6 (isolates Ac40 and Ac41), in which the isolates presented $100 \%$ similarity, and the same AME genes and aminoglycoside susceptibility profiles were observed [Supplementary data (Figure 1)].

Interestingly, some CR A. baumannii isolates presented different genotypes and susceptibility regarding aminoglycosides resistance, but exhibited $100 \%$ genotypic similarity to each other according to REP-PCR-typing, as observed in clusters A1 (Ac21 and Ac134), B1 (Ac30 and Ac54), C2 (Ac97, Ac100, and Ac139), and D4 (Ac50, Ac51, and Ac118) [Supplementary data (Figure 1)]. These discrepancies could be explained by the constant mobilization of AME genes, as they are often located in plasmids and class 1 integrons, suggesting that horizontal gene transfer plays an important role in the dissemination of aminoglycoside resistance in $A$. baumannii ${ }^{15}$.

It is also relevant to note that the dendrogram presents two characteristic profiles. The first one, represented by the groups A, $\mathrm{B}, \mathrm{C}$, and $\mathrm{E}$, that presents a similar and almost exclusive genotype of AME genes ( $a p h\left(3^{\prime}\right)-V I$ and occasionally $\left.a a c\left(6^{\prime}\right)-I b\right)$, with the majority of these members presenting resistance only to amikacin (Figure 1). The second profile, represented by group D, the most diverse group, presents a general common genotype of AME genes among its members ( $a a c\left(6^{\prime}\right)-I b, a a c(3)-I a$, and $a p h\left(3^{\prime}\right)$ $I a)$, and resistance to gentamicin and intermediate resistance to amikacin [Supplementary data (Figure 1)].

Another interesting finding is related to group $\mathrm{E}$, which presented lower similarity (46.7\%) to groups A, B, C, and D.
Its first isolate, Ac133 (cluster E1), was recovered on November 29 from the Emergency-ICU, where new severe inpatients are admitted; three days later, two isolates of the same E1 cluster (Ac130 and Ac138) were recovered from the General-ICU [Supplementary data (Figure 1)]. This may have occurred due to the introduction of a new cluster into the hospital, since their isolates were recovered only in the final period, and a rapid dissemination may have happened between the two ICUs.

In conclusion, we consider that a clonal dissemination of multidrug-resistant $A$. baumannii occurred within the institution. This finding emphasizes the need for improved infection control, such as isolation of colonized or infected patients and environmental disinfection interventions, to prevent further dissemination of $A$. baumannii in the institution.

\section{ACKNOWLEDGMENTS}

We would like to thank the microbiology laboratory staff of Hospital de Base de São José do Rio Preto for supplying and identifying the bacterial isolates.

\section{Conflict of Interest Statement}

The authors declare that there is no conflict of interest.

\section{Financial Support}

We would like to thank Coordenação de Aperfeiçoamento de Pessoal de Nível Superior (CAPES) for granting scholarships to the students involved in this research.

\section{REFERENCES}

1. Howard A, O'Donoghue M, Feeney A, Sleator RD. Acinetobacter baumannii: an emerging opportunistic pathogen. Virulence. 2012;3(3):243-50.

2. Neidell MJ, Cohen B, Furuya Y, Hill J, Jeon CY, Glied S, et al. Costs of healthcare- and community-associated infections with antimicrobial-resistant versus antimicrobial-susceptible organisms. Clin Infect Dis. 2012;55(6):807-15.

3. Viehman JA, Nguyen MH, Doi Y. Treatment options for carbapenem-resistant and extensively drug-resistant Acinetobacter baumannii infections. Drugs. 2014;74(12):1315-33. 
4. Asif M, Alvi IA, Rehman SU. Insight into Acinetobacter baumannii: pathogenesis, global resistance, mechanisms of resistance, treatment options, and alternative modalities. Infect Drug Resist. 2018;11:1249-60.

5. Akers KS, Chaney C, Barsoumian A, Beckius M, Zera W, Yu X, et al. Aminoglycoside resistance and susceptibility testing errors in Acinetobacter baumannii-calcoaceticus complex. J Clin Microbiol. 2010;48(4):1132-8.

6. Krawczyk B, Lewandowski K, Kur J. Comparative studies of the Acinetobacter genus and the species identification method based on the recA sequences. Mol Cell Probes. 2002;16(1):1-11.

7. Doi Y, Arakawa Y. 16S Ribosomal RNA Methylation: Emerging Resistance Mechanism against Aminoglycosides. Clin Infect Dis. 2007;45(1):88-94.

8. Čiginskienè A, Dambrauskienė A, Rello J, Adukauskienė D. Ventilator-Associated Pneumonia due to Drug-Resistant Acinetobacter baumannii: Risk Factors and Mortality Relation with Resistance Profiles, and Independent Predictors of In-Hospital Mortality. Medicina (B Aires) 2019;55:49.

9. Nowak J, Zander E, Stefanik D, Higgins PG, Roca I, Vila J, et al. High incidence of pandrug-resistant Acinetobacter baumannii isolates collected from patients with ventilator-associated pneumonia in Greece, Italy and Spain as part of the MagicBullet clinical trial. J Antimicrob Chemother. 2017;72(12):3277-82.

10. Yoon YK, Lee J, Ryu SY, Chang H-H, Choi WS, Yoon JH, et al. Clinical significance of multidrug-resistant Acinetobacter baumannii isolated from central venous catheter tip cultures in patients without concomitant bacteremia. Scand J Infect Dis 2013; 45: 900-906.

11. Turton JF, Woodford N, Glover J, Yarde S, Kaufmann ME, Pitt TL. Identification of Acinetobacter baumannii by Detection of the blaOXA-51-like Carbapenemase Gene Intrinsic to This Species. J Clin Microbiol 2006; 44: 2974-2976.

12. Pagano M, Barin J, Martins AF, Zavascki AP. High Endemic Rates of OXA-23-Producing Carbapenem-Resistant Acinetobacter baumannii Isolates Caused by the Persistence of Major Clones in Hospitals in a Brazilian City 5 Years After an Outbreak. Infect Control Hosp Epidemiol 2015; 36: 860-862.

13. Corvec S, Poirel L, Naas T, Drugeon H, Nordmann P. Genetics and expression of the carbapenem-hydrolyzing oxacillinase gene blaOXA-23 in Acinetobacter baumannii. Antimicrob Agents Chemother 2007; 51: 1530-3.

14. Sheikhalizadeh V, Hasani A, Ahangarzadeh Rezaee M, Rahmatiyamchi M, Hasani A, Ghotaslou R, et al. Comprehensive study to investigate the role of various aminoglycoside resistance mechanisms in clinical isolates of Acinetobacter baumannii. J Infect Chemother 2017; 23: 74-79.

15. Liu C-C, Tang CY, Chang K-C, Kuo H-Y, Liou M-L. A comparative study of class 1 integrons in Acinetobacter baumannii. Gene 2014; 544: $75-82$. 